\title{
Magnetic hard drives go atomic
}

Physicists demonstrate the first single-atom magnetic storage.

\section{Elizabeth Gibney}

08 March 2017

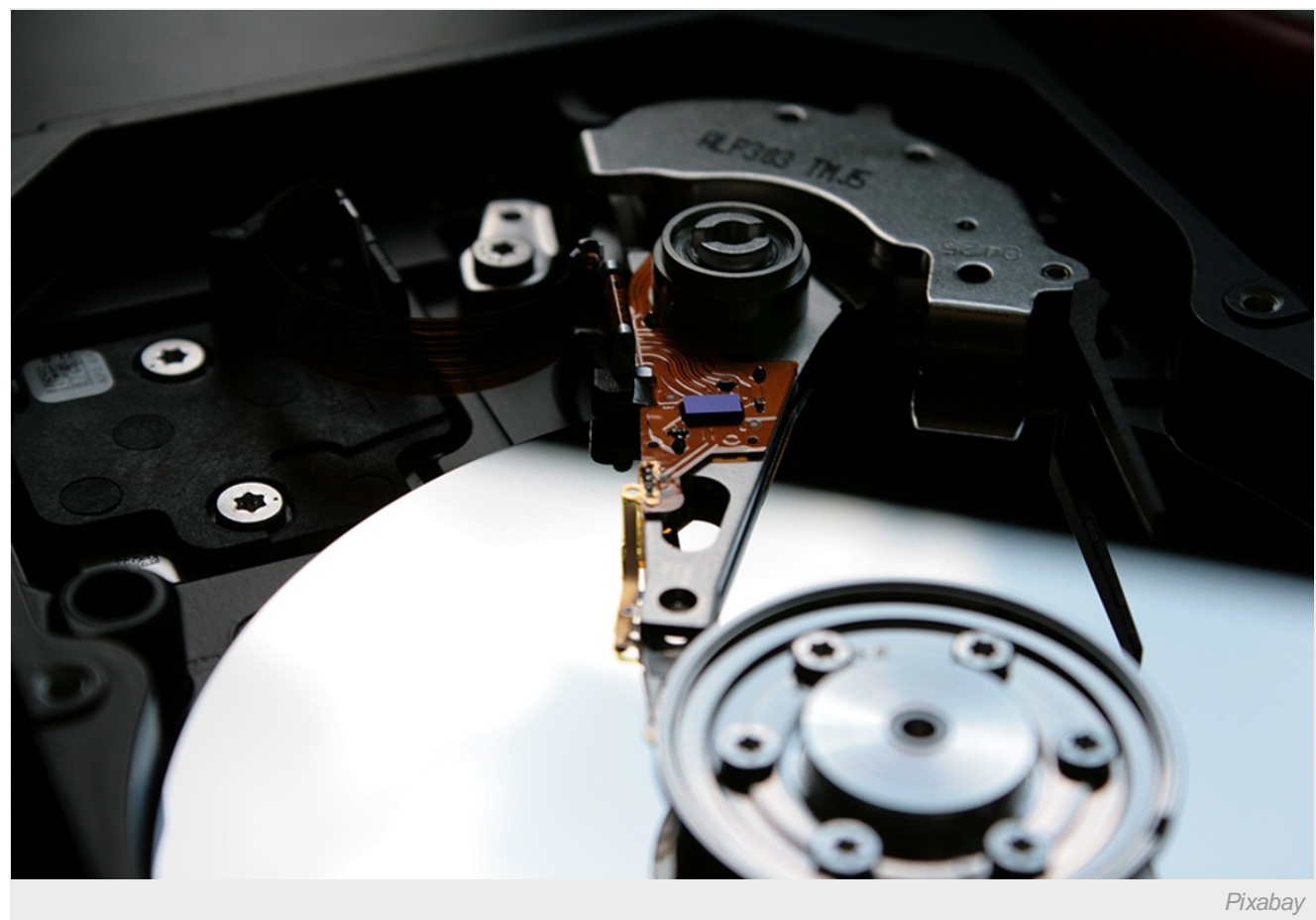

Existing hard drives use magnets made of about 1 million atoms to store a single bit of data.

Chop a magnet in two, and it becomes two smaller magnets. Slice again to make four. But the smaller magnets get, the more unstable they become; their magnetic fields tend to flip polarity from one moment to the next. Now, however, physicists have managed to create a stable magnet from a single atom.

The team, who published their work in Nature on 8 March $^{1}$, used their single-atom magnets to make an atomic hard drive. The rewritable device, made from 2 such magnets, is able to store just 2 bits of data, but scaled-up systems could increase hard-drive storage density by 1,000 times, says Fabian Natterer, a physicist at the Swiss Federal Institute of Technology (EPFL) in Lausanne, and author of the paper.

"It's a landmark achievement," says Sander Otte, a physicist at Delft University of Technology in the Netherlands. "Finally, magnetic stability has been demonstrated undeniably in a single atom."

Inside a regular hard drive is a disk split up into magnetized areas — each like a tiny bar magnet — the fields of which can point either up or down. Each direction represents a 1 or 0 - a unit of data known as a bit. The smaller the magnetized areas, the more densely data can be stored. But the magnetized regions must be stable, so that '1's and '0's inside the hard disk do not unintentionally switch

Current commercial bits comprise around 1 million atoms. But in experiments physicists have radically shrunk the number of atoms needed to store 1 bit - moving from 12 atoms in $2012^{2}$ to now just one. Natterer and his team used atoms of holmium, a rare-earth metal, sitting on a sheet of magnesium oxide, at a temperature below 5 kelvin.

Holmium is particularly suitable for single-atom storage because it has many unpaired electrons that create a strong magnetic field, and they sit in an orbit close to the atom's centre where they are shielded from the environment. This gives holmium both a large and stable field, says Natterer. But the shielding has a drawback: it makes the holmium notoriously difficult to interact with. And until now, many physicists doubted whether it was possible to reliably determine the atom's state. 


\section{Bits of data}

To write the data onto a single holmium atom, the team used a pulse of electric current from the magnetized tip of scanning tunnelling microscope, which could flip the orientation of the atom's field between a 0 or 1 . In tests the magnets proved stable, each retaining their data for several hours, with the team never seeing one flip unintentionally. They used the same microscope to read out the bit with different flows of current revealing the atom's magnetic state.

To further prove that the tip could reliably read the bit, the team — which included researchers from the technology company IBM — devised a second, indirect, read-out method. They used a neighbouring iron atom as a magnetic sensor, tuning it so that its electronic properties depended on the orientation of the two holmium atomic magnets in the 2-bit system. The method also allows the team to read out multiple bits at the same time, says Otte, making it more practical and less invasive than the microscope technique.

Using individual atoms as magnetic bits would radically increase the density of data storage, and Natterer says that his EPFL colleagues are working on ways to make large arrays of single-atom magnets. But the 2-bit system is still far from practical applications and well behind another kind of single-atom storage, which encodes data in atoms' positions, rather than in their magnetization, and has already built a 1-kilobyte (8,192-bit) rewritable data storage device.

One advantage of the magnetic system, however, is that it could be compatible with spintronics, says Otte. This emerging technology uses magnetic states not just to store data, but to move information around a computer in place of electric current, and would make for much more energy-efficient systems.

In the near term, physicists are more excited about studying the single-atom magnets. Natterer, for example, plans to observe three mini-magnets that are oriented so their fields are in competition with each other — so they continually flip. "You can now play around with these single-atom magnets, using them like Legos, to build up magnetic structures from scratch," he says.

Nature I doi:10.1038/nature.2017.21599

\section{References}

1. Natterer, F. et al. Nature http://dx.doi.org/10.1038/nature21371 (2017).

2. Loth, S., Baumann, S., Lutz, C. P., Eigler, D. M. \& Heinrich, A. J. Science 335, 196-199 (2012). 